\title{
Outcome of India Ink Corneal Tattooing in Disfigured Corneal Scars
}

\author{
Pallavi Joshi*, Sanjana P and Girish Budhrani \\ Department of Cornea, Ocular Surface and Refractive Surgery, Sankara Eye \\ Hospital, Bangalore, India \\ *Corresponding Author: Pallavi Joshi, Department of Cornea, Ocular Surface \\ and Refractive Surgery, Sankara Eye Hospital, Bangalore, India.
}

Received: March 03, 2020

Published: April 30, 2020

(C) All rights are reserved by Pallavi Joshi.,

et al.

\begin{abstract}
Aims: To study the outcome and safety of India ink corneal tattooing for disfigured corneal scars.

Settings and Design: This was a single-centre prospective interventional case series of patients who developed corneal scars with nil visual potential and underwent corneal tattooing from June 2017-September 2018 with a follow-up period of at least 6 months. Materials and Methods: 15 consecutive cases were enrolled for the study. All patients desired a cosmetic treatment for their corneal scars in blind eye. Complete ophthalmic examination including B scan and photographs of the patients' eyes were obtained. Chemical corneal tattooing was performed using Indian Ink Powder. Patients underwent either inlay, on lay or mixed tattooing and were followed up at the first, third and fifth post-operative day, at $3^{\text {rd }}$ month, $6^{\text {th }}$ month and 1 st year post-operatively.

Statistical Analysis Used: Descriptive statistics will be calculated initially. For continuous variables, the mean, standard deviation statistics will be calculated and proportion statistics for categorical data.

Results: Among the 15 cases, 7 (46\%) patients underwent intralamellar tattooing, 3(20\%) patients had onlay tattoo and 5 (33\%) patients underwent onlay with inlay tattoo. Patients who underwent onlay tattoo noted fading of tattoo at the end of 3 months. One year postoperatively, 12 cases ( $80 \%$ ) especially the inlay tattoo cases were more satisfied compared to onlay tattoo. All patients were asymptomatic with no evidence of any major ocular complications at the end of one year.

Conclusion: Chemical corneal tattooing is a simple, safe and an efficient technique yielding acceptable cosmetic results. It does not require expensive materials and offers a viable option in comparison to more extensive and invasive reconstructive ocular surgery. Cosmetic contact lenses being unsuitable for majority of cases owing to poor tolerance due to dust exposure and cost effectiveness tattooing of corneal opacities still has a role in cosmetic improvement in cases with unsightly corneal scars. Chemical corneal tattoo by using Indian ink as an alternative proves to give almost equivalent stable, satisfactory cosmetic result with high patient satisfaction in all cases and an improved quality of life.
\end{abstract}

Keywords: Corneal Opacity; Corneal Tattooing; Inlay Tattoo; India Ink

\section{Introduction}

Using pigment to mask unsightly corneal scars has been known for more than 2000 years [1]. It was first introduced in $160 \mathrm{AD}$ by a Greek physician - Galen; however, the procedure was used infrequently for centuries thereafter [1]. The process was not readily adopted until the early $20^{\text {th }}$ century, when Frederick H. Verhoe reported the safe and effective use of India ink to darken corneal scars [2].

Frequent indication in the past for corneal tattooing has been cosmetic-patients who present with a leucomatous cornea or corneal scar may have these aesthetically unpleasing conditions masked by the procedure. More recent being sectoral tattoo for iris defects, diplopia, intractable glare etc [3-5]. Alternative methods to improve the aesthetic appearance of disfigured corneas are cosmetic contact lenses, keratoplasty, wearing ocular prosthesis with or without an enucleation or evisceration [6].

Repeated penetrating keratoplasty (PK) may be associated with non-compliance to treatment, rejections or infections [7-10]. With contact lenses, intolerance frequently develops after prolonged use while wearing an ocular prosthesis over a scarred cornea often causes inflammation, infection and corneal erosion [7-10].

Hence tattooing of corneal opacities still has a role for the cosmetic improvement of unsightly corneal scars [7-10]. 
Classic corneal tattooing uses dyes that are applied to the anterior stroma following epithelial debridement or trans epithelial needle puncture [11]. The other method is wherein the dye is injected in a lamellar channel $[12,13]$.

\section{Aim of the Study}

Our study is aimed to assess the efficacy of India ink corneal tattooing to improve the ocular cosmetic appearance, to demonstrate its safety and to investigate its potential as an alternative to invasive reconstructive surgery for the cosmetic correction of disfigured corneas.

\section{Subjects and Methods}

This is a prospective, interventional case series conducted at Cornea and Anterior Segment Services of a tertiary care eye hospital in South India. The study has been approved by the Institutional Ethics Committee. The study was conducted in adherence to the tenets of the Declaration of Helsinki. The patients or the legal guardians (in case of children) signed an informed written consent for the surgical procedure, investigations, and use of clinical photographs.

All patients who underwent corneal tattooing between June 2017 - September 2018 were included.

Study inclusion criteria were superficial or deep corneal opacities, band keratopathy, and retro corneal opacities. Patients with phthisical eyes, thin corneas, corneal oedema (bullous keratopathy), anterior staphyloma and glaucoma were excluded from the study.

All patients enrolled had corneal scar or retro corneal opacity with no visual potential. Complete ophthalmic examination was performed including B scan ultrasound to exclude intraocular tumours. The depth of corneal opacity, corneal thickness, the presence and extent of band keratopathy and corneal vascularization was carefully assessed by Topcon SS OCT (Figure 1 and 2).

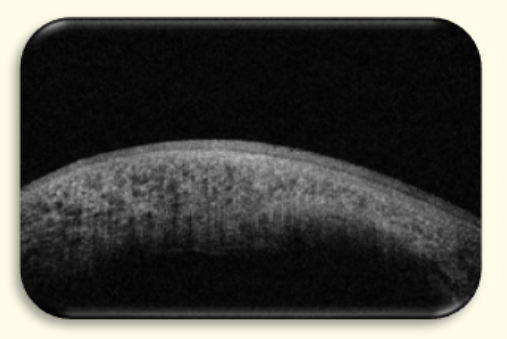

Figure 1: Total opacity - onlay tattoo.

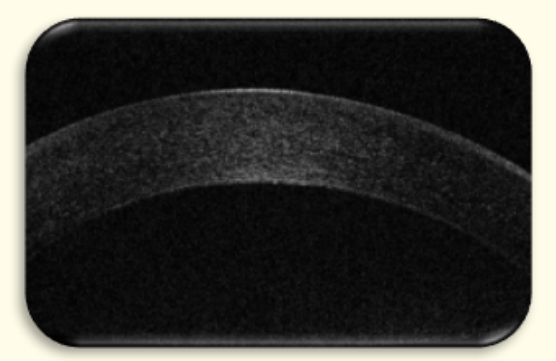

Figure 2: Deep stromal opacity - inlay tattoo.

Corneal tattooing was performed under general anaesthesia in children and local anaesthesia (retro bulbar) in adults.

Chemical corneal tattooing was performed using Indian Ink Powder (Mahatme laboratories).

Surgical techniques used were either Onlay tattooing, intra lamellar or combined. Onlay tattooing done using a $30 \mathrm{~g}$ needle, bevel down, to imbibe the ink into the anterior stroma. Thinning often associated with many corneal scars, tangential stab was done to avoid inadvertent perforation of the cornea. Inlay tattooing was done by creating the lamellar pocket with predepth 300/350 micron knife (Company) and using lamellar dissection followed by inserting with the tattoo material in two or three layers in the interface, incision closed with suture.

Postoperatively, topical steroid antibiotics combination (dexamethasone sodium $0.1 \%+$ chloramphenicol $0.5 \%$ Dexoren $S^{\circ}$, Indoco Remedies ltd.) four times a day and a cycloplegic (Homatropine $2 \%$, Homide ${ }^{\circ}$, Indoco Remedies ltd) two times a day. Patients were followed up on the first, third and fifth post-operative day, at $3^{\text {rd }}$ month, $6^{\text {th }}$ month and $1^{\text {st }}$ year post-operatively.

\section{Statistical analysis}

Statistical analysis was carried out using SPSS statistical software (SPSS version 21. Inc., Chicago, IL, USA). All descriptive parameters were noted in the form of mean and standard deviation if the data were parametric or in the form of median if the data were nonparametric.

\section{Results}

During the period of study fifteen eyes of fifteen patients met the inclusion criteria. Out of fifteen patients 11 were adults (mean age: 29.1 years, range: 20 - 42 years) and 4 were children (mean age: 8.1 years, range: 5 - 16 years). Males were more common than females (70\%). Among the 15 cases, 7 (46\%) patients underwent intralamellar tattooing, 3(20\%) patients had onlay tattoo done and $5(33 \%)$ patients underwent onlay with inlay tattoo (Figure 3 and 
4). Patients who underwent onlay tattoo had thinner and irregular cornea hence inlay not attempted (Figure 5).

3 cases had superficial corneal opacities (20\%), 7 had deep
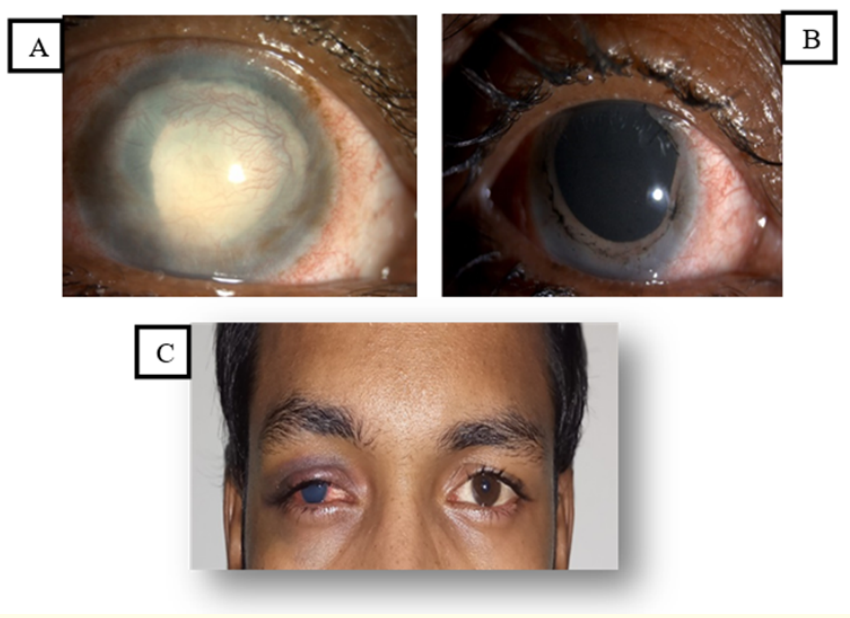

Figure 3: A: Deep stromal opacity B: Inlay tattoo

C: Portrait of same patient.
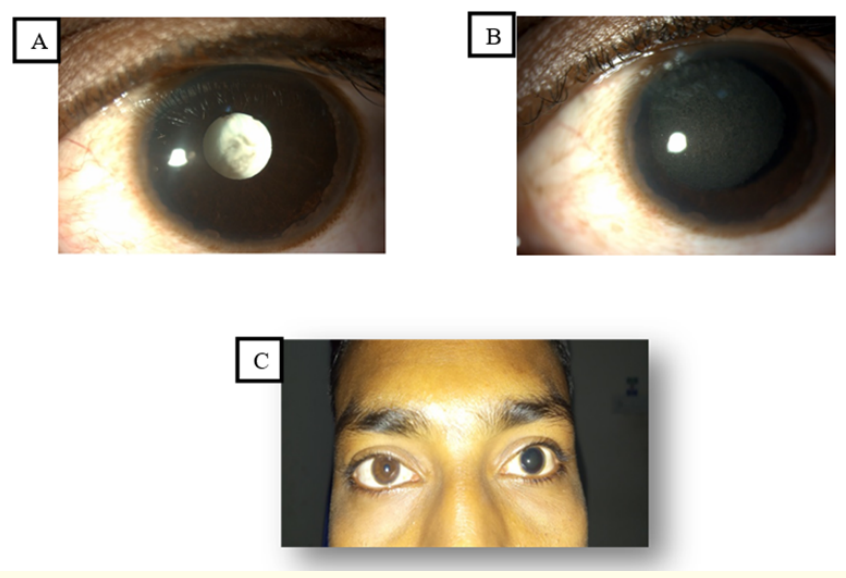

Figure 4: A: Absorbed Cataract with closed funnel RD B: inlay tattoo C: Portrait of same patient.

corneal opacities cases (46.6\%), 1 case cataract with no visual potential (6.6\%) and 4 cases presented with multiple failed graft (26.8\%) (Table 1).

Patient were followed up on regular time intervals and one year postoperatively, (80\%) 12 cases specially the inlay tattoo cases were more satisfied compared to onlay tattoo (Figure 6).

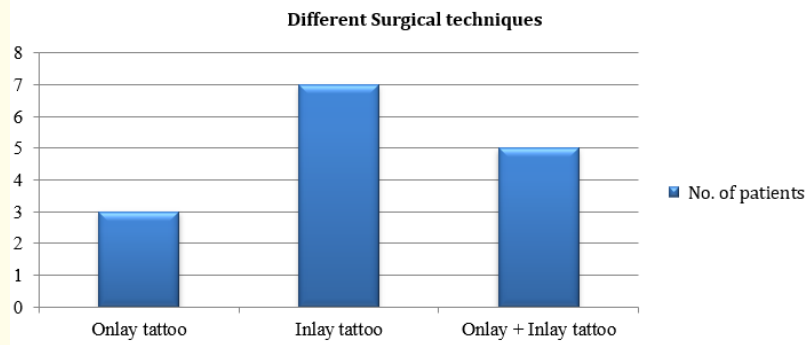

Figure 5: Cosmetic corneal tattoo: surgical technique.

\begin{tabular}{|c|c|c|}
\hline Sl. No. & Diagnosis & Eyes \\
\hline 1 & Post trauma & 2 \\
\hline 2 & Congenital opacity & 2 \\
\hline 3 & Post corneal ulcer & 3 \\
\hline 4 & Post failed graft & 4 \\
\hline 5 & Congenital cataract with RD & 1 \\
\hline 6 & Corneal opacity with Anterior staphyloma & 3 \\
\hline
\end{tabular}

Table 1: Diagnosis in recruited patients for corneal tattooing.

Grade of satisfaction of eyes- No. of patients

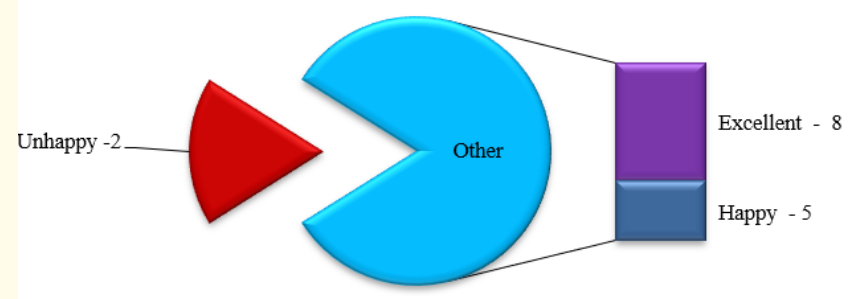

Figure 6: Percentage of patient satisfaction.

During the post-operative follow up, 8 patients complained of a moderate foreign body sensation and exhibited a conjunctival injection which corresponded to the surgically induced corneal epithelial defect and chemical reaction (53\%) for 2 days. The pigment density after an initial loss was nearly stabilized, the pigmentation was uniform. Majority of the cases 13 (86\%) did not require tattooing more than once, if the initial tattooing was satisfactory. Minimal pigment loss was observed in 4 cases (26\%) of which 2 (13\%) underwent repeat onlay after 3 months.

Corneal infection/corneal erosion were not noted in any case. Corneal melt (undergone inlay tattoo with epikeratoplasty) was noted in one patient (secondary to trauma). 
All patients were asymptomatic with no evidence of any ocular complications at the end of one year.

\section{Discussion}

Disfiguring corneal opacities may alter the personal self-confidence and affect badly both social lives and quality of life. Along these lines, we ought to consider cosmetic repair as an essential line of treatment of corneal opacities in such cases.

Tattooing of cornea is one of the options offered to patients with corneal opacities. Printed contact lenses, enucleation, or evisceration with orbital prosthesis and penetrating keratoplasty are some of the other options [6-10].

Chemical tattooing has proved to be an efficient and a simple technique [14]. It is a safe surgical procedure that does not require expensive materials and offers a viable option to avoid more extensive and invasive reconstructive ocular surgery [14].

Chemical corneal tattooing by our technique gives a stable, satisfactory cosmetic result with high patient satisfaction in all cases and an improved quality of life. Various tattooing methods were used such as chemical dyeing with the use of gold or platinum chloride and nonmetallic tattooing with India ink, China ink, lamp black/soot, and other organic dyes [15-17].

Surgeons experimented with different combinations of such chemical products to obtain different shades [18]. the main problem affecting the outcome of previous corneal tattoo studies was the fading of colors, which made the results inconsistent over time [18].

In our study we show that intrastromal technique provides more patient satisfaction and durability in comparison to onlay. A histopathology confirmation of this has been done by Amesty., et al [19].

Among the different methods of corneal tattooing described during the last century, one of them was a chemical dye with gold or platinum chloride, a simple technique, mainly used as only technique [20].

Most of the studies have noted recurrent corneal erosion and fading of tattoo [20]. In our study even though fading was noted, only 2 cases needed repeat tattooing hence suggesting India ink to be more durable and cornea friendly.

There are many reports of introducing the ink intrastromally by either manually dissecting a lamellar corneal pocket/ lamellar flap dissection or using the Fertiliser to create a flap [21,22].
In our study we preferred intrastromal lamellar pocketing with predepth knife after pre-operative evaluation like ASOCT and pachymetry to avoid risk of perforation followed by ink insertion.

Homogeneous pigmentation, faster surgery with a better and less symptomatic postoperative recovery among patients are the advantages. The corneal surface being untouched, the staining is not exposed to the tear film hence less symptomatic post procedure.

Previous studies have correlated injury to basement membrane of corneal epithelium in onlay technique predisposing the eye to a condition akin to recurrent corneal erosions [23]. This fact is similar to our studies where patients had on and off mild redness post onlay technique.

The greater pigment density and its relative permanence is understandable as by the lamellar procedure, two surfaces are being tattooed simultaneously, the corneal bed and the inner surface of the lamellar flap, thus duplicating the amount of pigment deposited in the cornea. Larger the pigment initially deposited in cornea, the greater is the density of tattooing and more durable are the results $[19,23]$ similar to our cases with inlay intrastromal tattooing [24].

\section{Limitation of the Study}

Limitation of this study is the small number of cases included in comparison to other studies and the need for a long-term follow-up for patients treated with corneal tattooing even when satisfactory results are achieved in the short term.

\section{Conclusion}

To conclude India ink corneal tattoo used as described in this study achieves stable and durable results with good cosmetic satisfaction among patients, Corneal leucomas, leukocoria with nil visual potential are ideal patients and helps improve patient's quality of life. According to the results of this clinical study, corneal tattoo is a safe surgical procedure especially intralamellar technique with an easy learning curve. It does not require expensive materials and avoids more extensive and invasive reconstructive ocular procedures.

\section{Key Messages}

Corneal tattooing with India ink especially intra lamellar is cost effective, durable and cosmetically satisfactory in patients with corneal scars with nil visual prognosis in comparison to recurrent costs, special care with contact lens use or other invasive ocular surgeries for cosmesis. 


\section{Bibliography}

1. Dougan C., et al. "Seeing better through someone else's eyes". Brown University.

2. Verhoe FH. "India ink infiltration: a simple and effective substitute for corneal tattooing". Journal of the American Medical Association 17 (1917): 1420-1221.

3. Burris TE., et al. "Lamellar intrastromal corneal tattoo for treating iris defects (artificial iris)". Cornea 17 (1998): 169173.

4. Stone NM., et al. "Intractable diplopia: a new indication for corneal tattooing”. British Journal of Ophthalmology 92.1445 (2008): 561-562.

5. Ahn SJ., et al. "A case of superficial corneal tattooing for glare after trabeculectomy". Canadian Journal of Ophthalmology 44 (2009): e63.

6. Vander Velden-Samderubun EM., et al. "Corneal tattooing". Cornea (1995): 14-225e30.

7. Hallock GG. "Cosmetic trauma surgery". Plastic and Reconstructive Surgery 95 (1995): 380e15.

8. Kuzan Jr WM. "Plastic surgery". Journal of the American College of Surgeons 188 (1999): 171-177.

9. Hoeyberhs JL. "Fortnightly review: cosmetic surgery". British Medical Journal 318 (1999): 512-516.

10. Custer PL., et al. "Orbital implants in nucleation surgery. A report by the American Academy of Ophthalmology". Ophthalmologica 110 (2003): 2054-2061.

11. Pitz S., et al. "Corneal tattooing: an alternative treatment for disfiguring corneal scars". British Journal of Ophthalmology 86 (2002): 397-399.

12. GD Kymionis., et al. "Femtosecond-assisted anterior lamellar corneal staining tattooing in a blind eye with leukocoria". Cornea 28.2 (2009): 211-213.

13. H Alsmman Hassan and NG Abd Elhaliem Soliman. "Intrastromal injection of China painting ink in corneas of male rabbits: clinical and histological study". Journal of Ophthalmology (2016).

14. Gupta D and Broadway D. "Cost-effective tattooing: the use of sterile ink for corneal tattooing after complicated peripheral iridotomies: an alternative to expensive salts". Journal of Glaucoma 19.8 (2010): 566-567.
15. S Holth. "Revival of Galen's corneal staining with copper sulfate and tannine should be abandoned". American Journal of Ophthalmology 14 (1931): 378-379.

16. EM van der Velden Samderubun and JHC Kok. "Dermatography as a modern treatment for coloring leukoma cornea". Cornea 13.4 (1994): 349-353.

17. JN Roy. "Tattooing of the cornea 1938". Canadian Medical Association Journal 39.5 (1938): 436-438.

18. Gifford SR and Steinberg A. "Gold and silver impregnation of cornea for cosmetic purposes". American Journal of Ophthalmology 10 (1927): 240-247.

19. MA Amesty., et al. "Tolerance of micronized mineral pigments for intrastromal keratopigmentation: a histopathology and immunopathology experimental study". Cornea 35.9 (2016): 1199-1205.

20. Duggan JN and Nanavati BP. "Tattooing of corneal opacity with gold and platinum chloride". British Journal of Ophthalmology 20 (1936): 419e25.

21. Fogla R., et al. "Microkeratome-assisted corneal tattooing: a case report”. Cornea 29 (2010): 446-448.

22. Kim C., et al. "New surgical strategy for corneal tattooing using a femtosecond laser". Cornea 28 (2009): 80-84.

23. Panda A., et al. "Corneal tattooing-experiences with "lamellar pocket procedure". Indian Journal of Ophthalmology 32 (1984): 408-411.

24. C Kim., et al. "Five-year results of corneal tattooing for cosmetic repair in disfigured eyes”. Cornea 30.10 (2011): 1135-1139.

\section{Assets from publication with us}

- Prompt Acknowledgement after receiving the article

- Thorough Double blinded peer review

- Rapid Publication

- Issue of Publication Certificate

- High visibility of your Published work

Website: www.actascientific.com/

Submit Article: www.actascientific.com/submission.php

Email us: editor@actascientific.com

Contact us: +919182824667 